\title{
CNS and systemic inflammation underlying neuropsychiatric symptoms and related cognitive decline in older people.
}

Christopher Clark ( $\nabla$ christopher.clark@irem.uzh.ch )

Universitat Zurich https://orcid.org/0000-0001-7210-7702

Jonas Richiardi

Université de Lausanne

Bénédicte Maréchal

Siemens Healthcare $\mathrm{GmbH}$

Gene L. Bowman

Nestle Institute of Health Sciences SA

Loïc Dayon

Nestle Institute of Health Sciences SA

Julius Popp

UniversitatsSpital Zurich

Research

Keywords: Neuroinflammation, neuropsychiatric symptoms; biomarkers, CSF, serum, cognitive decline

Posted Date: May 9th, 2020

DOl: https://doi.org/10.21203/rs.3.rs-25972/v1

License: (9) (i) This work is licensed under a Creative Commons Attribution 4.0 International License. Read Full License 


\section{Abstract}

BACKGROUND Neuroinflammation may contribute to psychiatric symptoms in older people, in particular in the context of Alzheimer's disease (AD). Here, our objective was to determine systemic and central nervous system (CNS) inflammatory signatures associated with neuropsychiatric symptoms (NPS) in older subjects, and investigate their relationships with AD pathology and cognitive decline.

METHODS We quantified a panel of inflammatory markers in both cerebrospinal fluid (CSF) and circulating blood serum in elderly subjects with normal cognition or with beginning cognitive decline. We further performed a comprehensive clinical assessment including longitudinal cognitive and neuropsychiatric evaluations and measured CSF biomarkers of core AD pathology. Multivariate analysis selected CSF and serum neuroinflammatory molecules associated with the presence of overall NPS and specific symptoms.

RESULTS The presence of NPS was associated with distinct inflammatory markers profiles involving soluble intracellular cell adhesion molecule-1 (sICAM-1), C-reactive protein (CRP), Interleukin (IL) -8 and $10 \mathrm{kDa}$ interferon- $\gamma$-induced protein (IP-10) in CSF; and Eotaxin-3, IL-6 and CRP in serum. Further analysis identified specific inflammatory marker signatures associated with anxiety, depression and disinhibition. Presenting NPS was associated with subsequent cognitive decline and this association was mediated by CSF SICAM-1.

CONCLUSIONS These results suggest that NPS in older people are associated with distinct systemic and CNS inflammatory processes. Neuroinflammation may explain the link between NPS and more rapid clinical disease progression.

\section{Background:}

Neuropsychiatric symptoms (NPS) are behavioral, emotional and psychological non-cognitive disturbances caused by cerebral pathology (1). NPS are frequent among patients with central nervous system (CNS) pathologies (2), including Alzheimer's disease (AD) $(3,4)$, and often precede dementia (57) and mild cognitive impairment (8). NPS are also associated with more rapid cognitive and functional decline $(4,8-10)$, through unknown pathogenic mechanisms $(11,12)$. In addition, atrophy in brain regions correlated with NPS (13) is associated with more rapid disease progression in AD (14).

Various neuropsychiatric disorders are linked with inflammation. Indeed, the co-occurrence of anxiety, depression, and apathy in relation to systemic inflammation (i.e. sickness behavior) is caused by the CNS immune response activated through cytokines(15). In animal models, this is associated with increased neurodegeneration (16-18). In humans, systemic and CNS inflammation have been associated with NPS (19-21), cerebrovascular and neurodegeneration processes, and cognitive decline (22-25). In the context of $A D$, neuroinflammation markers present in peripheral blood could indicate cerebral pathology $(22,26)$ and be associated with disease severity (27). However, the inflammatory processes and mechanisms associated with NPS and their contribution to the clinical course remain unknown. 
This study aims to identify systemic and CNS inflammatory patterns associated with NPS in older subjects while considering relationships with cognitive impairment and core AD pathology. We will also explore the relationship between the identified inflammatory markers and brain volumetric data to determine if NPS related neuroinflammation is associated with specific changes in regional volumetry. Finally, we will address the longitudinal relationships between NPS, inflammation and cognitive decline.

\section{Methods:}

\subsection{Study population:}

120 community dwelling individuals, aged 55 or older, were enrolled into a brain aging study conducted in the Department of Psychiatry and the Department of Clinical Neurosciences, University Hospital of Lausanne, Switzerland. They were recruited among memory clinic outpatients or through advertisement. An overall clinical, neurological and comprehensive neuropsychological assessment was performed, and candidates with neurological or psychiatric affections that may interfere with cognitive diseases, or medical conditions such as acute infections were excluded as described in (22). Clinical and neuropsychological follow-up evaluations were performed at 18 and 36 months using the same methods and tests whenever possible.

\subsection{Study procedures}

\subsubsection{Neuropsychological assessments:}

Along with the clinical examination, the Neuropsychiatric Inventory questionnaire (NPI-Q) (28) was administered to assess neuropsychiatric symptoms. Twelve categories, ten behavioral and two neurovegetative (Night-time behavior and Appetite/Eating), were scored for their severity ranging from 0 to 3. Total NPI-Q score was obtained by adding the twelve scores. Participants with a total NPI-Q score of 0 and with no history or evidence of NPS were considered controls. We also collected Clinical Dementia Rating (CDR), CDR sum of boxes (CDR-SoB), and Mini-Mental State Examination (MMSE). Cognitive state was classified using CDR.

\subsubsection{Biochemical sample collection and handling:}

Lumbar and venous punctures conducted during the same visit yielding $10-12 \mathrm{ml}$ of CSF and $40 \mathrm{ml}$ of

blood respectively were performed after an overnight fast in the memory center, spun down at $4{ }^{\circ} \mathrm{C}$, immediately aliquoted, and snap frozen at $-80^{\circ} \mathrm{C}$ until assayed (22). Study personnel blinded to clinical data performed biochemical and genetic analyses.

\subsubsection{Inflammatory marker measurements:}

Quantitative analysis of 38 neuroinflammatory biomarkers in CSF or serum was achieved using a sandwich immunoassay (Meso Scale Diagnostics, Rockville, USA) as previously described (29). Biomarkers with more than $5 \%$ missing data or below-level-of quantification were filtered out, resulting in 
a selection of 28 analytes. These included basic fibroblast growth factor, C-reactive protein (CRP), eotaxin-1, eotaxin-3, interferon-y (IFN-y), interleukin-12, interleukin-15 (IL-15), interleukin-16 (IL-16), interleukin-6 (IL-6), interleukin-7, interleukin-8 (IL-8), 10 kDa interferon-y-induced protein (IP-10), monocyte chemoattractant protein 1 (MCP-1), monocyte chemoattractant protein 4 (MCP-4), macrophage-derived chemokine (MDC), macrophage inflammatory protein 1a (MIP-1a), macrophage inflammatory protein $1 \beta$, phosphorylated insulin-like growth factor-1 receptor, serum amyloid A, soluble fms-like tyrosine kinase-1 (sFLT-1), soluble intercellular adhesion molecule-1 (sICAM-1), circulating vascular cell adhesion molecule1 (sVCAM-1), thymus and activation-regulated chemokine (TARC), angiopoietin-1 receptor, tumor necrosis factor-a, vascular endothelial growth factor (VEGF), vascular endothelial growth factor $\mathrm{C}$ and vascular endothelial growth factor D precursor (VEGF-D).

\subsubsection{Biochemical measures:}

The CSF albumin index (Qalb) of blood-brain barrier (BBB) impairment along with the APOE genotype were determined as previously described (29).

\subsubsection{Cerebrospinal fluid AD biomarkers:}

CSF beta-amyloid 1-42 (Aß1-42), total-tau (Tau), and tau phosphorylated at threonine 181 (pTau181) concentrations were measured using commercially available ELISA kits (Fujirebio, Gent, Belgium). A $\mathrm{pTau} / \mathrm{A} \beta_{1-42}$ ratio $>0.0779$ was defined as an AD CSF profile (22).

\subsubsection{Volumetric measurements:}

All participants underwent a magnetic resonance imaging scan on a 3T MRI system (MAGNETOM Prisma ${ }^{\text {fit }}$, Siemens Healthcare, Erlangen, Germany) with a 32-channel head coil. Acquisitions followed the ADNI2 MRI protocol (30). Images were then segmented with the MorphoBox prototype algorithm (31); briefly, this registers subject data to an internal template established by consensus segmentation of neuroradiologists, applies bias field correction with a 4-tissue class (gray matter (GM), white matter (WM), cerebro-spinal fluid (CSF), non-brain) Gaussian mixture model, performs skull-stripping, classifies brain tissue into 5 classes (ventricular CSF, sulcal CSF, cortical GM, deep GM, and WM) via variational expectation-maximisation yielding 5 posterior probability maps, and provides regional volume estimates by summing up these probabilities within template regions. Quality was checked using established automated image quality (32) and segmentation quality metrics (31), and no images were rejected. We analyzed 18 brain structures and features including: amygdala, caudate nucleus, cerebellum, cortical grey matter, grey matter, hippocampus, insula, medulla oblongata, mesencephalon, pallidum, pons, putamen, thalamus, 3rd ventricle, 4th ventricle, total ventricular volume, white matter, white matter abnormalities. This regional volumetric data was normalized by total intracranial volume (defined as the sum of gray matter, white matter and CSF).

\subsection{Data preparation and transformation:}

Before analysis, outliers (i.e. data points that exceeded the cutoff value of mean $\pm 3 \times \mathrm{SD}$ ) were replaced by the cutoff value. Biomarker data was $\log _{n}$-transformed prior to correlation and regression analyses to 
approach Gaussian distribution. For participants displaying a positive NPI-Q score, we computed an NPI severity variable by $\log _{\mathrm{n}}$-transforming NPI-Q score.

\subsection{Statistical and analytical approaches:}

Descriptive statistics for the cohort were performed using t-tests comparing NPI-Q and control group for continuous variables and Chi-square tests for categorical variables. Box plots and t-tests compared biomarker distribution and concentration between groups. Correlations implicating neuropsychological measures were assessed with Spearman's rho. Benjamini-Hochberg correction of $P$ value for multiple testing was then applied using a false-discovery rate of 0.1 . Independence of variables used in regression models was tested with variance inflation factor (VIF). No variable entered in these models had VIF above 7 , with a majority below 3 , thus absence of multicollinearity was assumed. Considering our effect sizes $(>0.7)$, the size of this cohort results in a statistical power of 0.9 or more. Statistical data analysis was performed with IBM SPSS Statistics software version 25.

\subsubsection{Statistical modelling:}

To select neuroinflammatory marker combinations in CSF or serum associated with NPS, we used binary regression models with $\mathrm{NPI}-\mathrm{Q}>0$ or $\mathrm{NPI}-\mathrm{Q}=0$ as dependent variable while entering all CSF or serum markers. We explored the following confounders: cognitive status (CDR $=0$ or CDR $>0$ ), CSF AD biomarker profiles (pTau/A $\beta_{1-42}$ ratio) and BBB impairment (Qalb) by entering them into the model before considering inflammatory marker concentrations. NPI severity score was then correlated to CSF or serum inflammation markers using linear regression with similar corrections. Associations of inflammatory markers with individual symptom category impairment (NPI-Q $>0$ for each individual category) was assessed using a binary logistic regression model corrected for cognitive status as above. All models used a forward selection method based on the significance of the score statistic. To construct a reference model, we used a separate binary logistic regression model with NPI-Q $>0$ or NPI-Q = 0 as dependent variable while using available demographic and clinical measures, including APOE\&4 status, sex, age, years of study and cognitive status to predict the occurrence of NPS. A receiver operating characteristic (ROC) curve and area under the curve (AUC) were computed for this model. CSF markers associated with NPS selected by the above models where then added to this model and ROC curves and AUCs were compared using the DeLong method. Interaction between AD pathology and neuroinflamamtion was tested using binary and linear regression models with either: CSF AD biomarkers $\times$ neuroinflammatory biomarkers versus NPS or NPI-Q score $\times$ CSF AD biomarkers versus CSF biomarkers, respectively. Associations between morphometric data and CSF neuroinflammatory markers were assessed using linear regression models with stepwise selection method and individual CSF molecule concentration as dependent variables entering all volumetric measurements. Linear regression was used to test associations between CSF neuroinflammatory markers and cognitive decline computed by change in CDR-SoB over time corrected for initial CDR-SoB assessment. Binary logistic regression with cognitive decline as dependent variable was used to test the associations of NPI-Q score, sICAM-1 concentration and NPI-Q × sICAM-1 concentration with cognitive decline at 18 and 36 months. In order to verify for 
possible overfitting of the above logistic regression models, we used the Hosmer-Lemeshow test for goodness-of-fit. Models with a Hosmer-Lemeshow chi-squared value yielding a P-value $>0.05$ were rejected and the previous iteration was considered instead.

\section{Results:}

\subsection{Characteristics of the cohort}

Serum, CSF, neuropsychiatric inventory and cognitive measures were available in 87 participants. Demographics, clinical and biological characteristics of participants by group with or without NPS are given in Table 1. The frequency of the different individual symptoms measured by the NPI-Q is presented in Table 2. The measured concentrations of individual CSF and serum neuroinflammatory markers are shown in Supplementary Tables 1 and 2 respectively. Longitudinal distribution at baseline, at 18- and at 36- months follow-up visits, of both cognitive status ( $C D R=0$ or $C D R>0)$ and the occurrence of NPS (NPI$\mathrm{Q}=0$ or NPI-Q $>0$ ) is shown in Supplementary Table 3. 
Table 1

Clinical and biochemical characteristics of the cohort.

\begin{tabular}{|c|c|c|c|}
\hline Clinical characteristic & $\begin{array}{l}N P I-Q=0 \\
(n=48)\end{array}$ & $\begin{array}{l}N P I-Q>0 \\
(n=39)\end{array}$ & $P$ value \\
\hline Sex, Female, n (\%) & $34(58.6)$ & $24(41.4)$ & 0.360 \\
\hline Age, yr, mean \pm SD & $68.19 \pm 8.01$ & $71.82 \pm 6.1$ & 0.022 \\
\hline Years of study \pm SD & $12.9 \pm 2.3$ & $12.1 \pm 2.8$ & 0.115 \\
\hline \multicolumn{4}{|l|}{ Cognitive function } \\
\hline$C D R$, mean $\pm S D$ & $0.2 \pm 0.3$ & $0.4 \pm 0.3$ & $<0.001$ \\
\hline$C D R S O B$, mean $\pm S D$ & $0.34 \pm 1.03$ & $1.71 \pm 2.04$ & $<0.001$ \\
\hline$M M S E, \pm S D$ & $28.4 \pm 1.92$ & $26.03 \pm 3.44$ & $<0.001$ \\
\hline \multicolumn{4}{|l|}{ AD CSF biomarkers } \\
\hline$A \beta 1-42, \mathrm{pg} / \mathrm{ml}$, mean $\pm S D$ & $939.72 \pm 235.74$ & $725.32 \pm 257.72$ & $<0.001$ \\
\hline Tau, pg/ml, mean $\pm S D$ & $240.08 \pm 113.76$ & $507.56 \pm 354.85$ & $<0.001$ \\
\hline pTau $181, \mathrm{pg} / \mathrm{ml}$, mean $\pm S D$ & $50.96 \pm 21.87$ & $75.32 \pm 47.42$ & 0.002 \\
\hline$p T a u / A \beta$ ratio, mean $\pm S D$ & $0.06 \pm 0.03$ & $0.13 \pm 0.12$ & $<0.001$ \\
\hline \multicolumn{4}{|l|}{ Biochemical measures } \\
\hline ApoEc4, $n(\% \varepsilon 4)$ & $8(27.6)$ & $21(72.4)$ & $<0.001$ \\
\hline$Q A l b$, mean $\pm S D$ & $5.02 \pm 1.71$ & $6.95 \pm 2.62$ & $<0.001$ \\
\hline \multicolumn{4}{|l|}{ Neuropsychiatric status } \\
\hline$N P /$ score, mean $\pm S D$ & $0.0 \pm 0.0$ & $5.74 \pm 5.26$ & $<0.001$ \\
\hline
\end{tabular}

\section{Table 1}

$P$ value represents result of t-test comparing groups with and without neuropsychiatric symptoms (NPI-Q $>0$ and $\mathrm{NPI}-\mathrm{Q}=0$, respectively) for continuous variables and Chi-square test for categorical variables (male/female frequency and APOEE 4 distribution). CDR, Clinical Dementia Rating; CDR-SoB, Clinical Dementia Rating Sum of Boxes; MMSE, Mini-Mental State Exam; A 1 1-42, beta-amyloid 1-42; Tau, totalTau; pTau, tau phosphorylated at threonine 181; Qalb, Quotient albumin or CSF albumin index, NPI-Q, Neuropsychiatric inventory questionnaire score.

\subsection{Neuroinflammatory signatures associated with the presence of NPS}


We found 5 markers in CSF (Fig. 1A) and 3 in serum (Fig. 1B) displaying significantly different concentrations between participants with $(\mathrm{NPI}-\mathrm{Q}>0)$ orwithout NPS $(\mathrm{NPI}-\mathrm{Q}=0)$. Overall NPI-Q score was positively correlated with the concentrations of sICAM-1, sVCAM-1, sFLT-1, IL-8 and IL-15, and MCP-1 and MCP-4 in CSF in the whole cohort (Fig. 1C). In serum, the concentration of MIP-1 a showed a significant positive correlation with NPI-Q, while for CRP and VEGF this correlation was negative (Fig. 1D).

Table 2

Neuropsychiatric inventory questionnaire (NPI-Q) scores within the study cohort.

\begin{tabular}{|lllll|}
\hline $\begin{array}{l}\text { Neuropsychiatric symptoms } \\
\text { distribution }\end{array}$ & $\begin{array}{l}\text { Mean score } \mathbf{S D} \\
\text { NPI-Q score }\end{array}$ & $\begin{array}{l}\text { Controls, } \mathbf{n} \\
(\%)\end{array}$ & $\begin{array}{l}\text { Symptoms, } \mathbf{n} \\
(\%)\end{array}$ & $\begin{array}{l}\text { CDR }=\mathbf{0}, \mathbf{n} \\
(\%)\end{array}$ \\
\hline Delusions & $2.57 \pm 4.52$ & $48(55.2)$ & $39(44.8)$ & $11(12.6)$ \\
\hline Hallucinations & $0.09 \pm 0.45$ & $81(95.3)$ & $4(4.7)$ & $1(1.1)$ \\
\hline Agitation & $0.06 \pm 0.32$ & $82(96.5)$ & $3(3.5)$ & $1(1.1)$ \\
\hline Depression/Dysphoria & $0.22 \pm 0.56$ & $71(83.5)$ & $14(16.5)$ & $2(2.3)$ \\
\hline Anxiety & $0.26 \pm 0.62$ & $70(82.4)$ & $15(17.6)$ & $5(5.7)$ \\
\hline Apathy & $0.48 \pm 0.85$ & $60(70.6)$ & $25(29.4)$ & $7(8)$ \\
\hline Irritability & $0.07 \pm 0.34$ & $81(95.3)$ & $4(4.7)$ & $2(2.3)$ \\
\hline Euphoria & $0.29 \pm 0.65$ & $68(80)$ & $17(20)$ & $2(2.3)$ \\
\hline Disinhibition & $0.08 \pm 0.41$ & $81(95.3)$ & $4(4.7)$ & $1(1.1)$ \\
\hline Ab motor behavior & $0.2 \pm 0.55$ & $73(85.9)$ & $12(14.1)$ & $3(3.4)$ \\
\hline Night-time behavior & $0.09 \pm 0.43$ & $80(94.1)$ & $5(5.9)$ & $0(0)$ \\
\hline Appetite/Eating & $0.27 \pm 0.63$ & $69(81.2)$ & $16(18.8)$ & $6(6.9)$ \\
\hline
\end{tabular}

\section{Table 2}

NPI-Q scores within the whole cohort for total score and individual categories. "Controls" represents cases were NPI-Q score is 0 for the overall or categorical score $=0$. All other cases are classified as "Symptoms". The percentage of cognitively healthy individuals within the whole cohort suffering from neuropsychiatric symptoms overall and in individual categories is also shown (CDR $=0)$. CDR, Clinical Dementia Rating; $A b$ motor behaviour, Aberrant motor behaviour.

Using binary regression models, we identified a combination of 4 neuroinflammatory markers in CSF that best predicted the occurrence of NPS: CRP, IP-10, sICAM-1 and IL-8 (Fig. 2A). In serum, this combination was: Eotaxin-3, IL-6 and CRP (Fig. 2B). Because we observed positive NPI-Q scores in the absence of cognitive impairment (Table 2), we refined these models by correcting them for baseline cognitive status. This did not change the combination of CSF inflammation markers associated with the occurrence of 
NPS, but in serum only CRP remained associated with a positive NPI-Q score (Fig. 2A and 2B). To investigate the effect of $A D$ pathology on these associations, we corrected our model for the presence or absence of $A D$ pathology at baseline. Once again, this did not change the CSF neuroinflammatory marker associations, only adding the CSF signature of AD pathology to the model (Fig. 2A). In serum, VEGF-D was added to the model together with the CSF biomarkers (Fig. 2B). We also investigated the effects of blood-brain barrier (BBB) impairment on this association by further correcting our model for Qalb. In this context the CSF inflammatory markers associated with the occurrence of NPS remained the same although the association of IL-8 with NPI-Q was no longer significant (Fig. 2A, P value $=.059$ ). In serum, MIP-1 a, VEGF and Qalb itself were associated with the occurrence of NPS (Fig. 2B).

When added to a reference model built using $A P O E$ status, Sex, Age, Years of study and Presence/Absence of cognitive impairment (Supplementary Table 4); sICAM-1, IP-10, IL-8 and CRP together significantly contributed to improve prediction of NPS (Supplementary Fig. 1, AUC $=0.908, P$ value $=0.0058$ )

Severity of NPS was only associated with CSF TARC levels when accounting for all conditions mentioned above (Fig. 2C). In serum, only IL- 6 was always associated with NPI-Q severity, whereas sICAM-1, IFN- $\gamma$, MCP-4 where associated with the severity of NPI-Q in the uncorrected model with the addition of IL-16 in the CSF AD biomarkers and BBB permeability models (Fig. 2D).

\subsection{Neuroinflammatory markers associated with individual neuropsychiatric syndromes}

We also evaluated the association of neuroinflammatory marker concentrations in both CSF and serum with the occurrence of each individual syndrome measured by NPI-Q (Table 3). Associations of inflammatory markers or combinations of markers in both CSF and serum emerged for nine out of twelve symptom categories. In all cases, CSF and serum associations were distinct. In CSF, sICAM-1 was overrepresented, with strong associations with Depression, Anxiety and Disinhibition. In serum, we found associations in 5 out of 12 symptoms and the strongest association observed was between VEGF-D and Depression (Table 3).Other molecules associated with the occurrence of overall NPS or their severity, such as MCP-4,CRP, sVCAM-1, VEGF or VEGF-D also displayed (albeit weaker) associations with individual symptoms. Conversely, we also found associations of IL-12 and MDC with Irritability and Night-time behavior, respectively even though these molecules were not associated to NPS in general. 
Table 3

Binary logistic regression revealing associations of neuroinflammatory markers with Neuropsychiatric inventory questionnaire (NPI-Q) sub-categories.

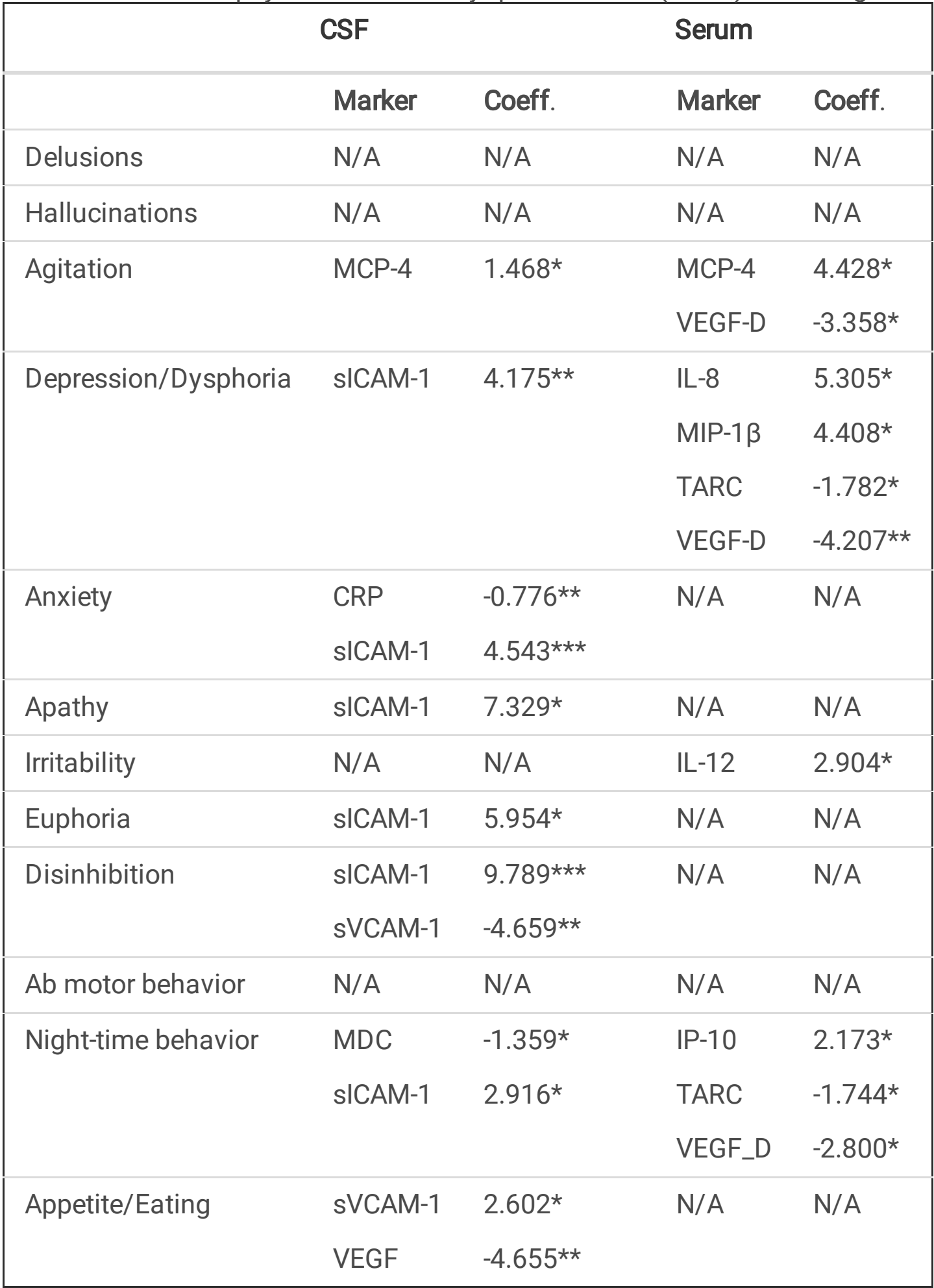

\subsection{Interaction between neuroinflammation, NPS and AD pathology}

Since the CSF concentrations of CRP, IP-10, sICAM-1, and AD status at baseline were associated with a positive NPI-Q score, we sought to test whether the interaction between neuroinflammation markers and the presence of AD pathology was associated with NPS. Using regression models with interaction 
variables we found that only the interaction between $A D$ pathology and sICAM-1 was significantly associated with the occurrence of NPS (Nagelkerke $\mathrm{R}^{2}=0.363$; coefficient $=3.386$; $P$ value $=.027$ ). The converse interaction model, i.e. the interaction between NPI-Q score and CSF AD biomarkers showed no significant association with CSF neuroinflammatory markers.

\subsection{Neuroinflammatory markers and brain morphometry}

We next explored the associations between the NPS-relevant neuroinflammatory CSF markers (Fig. 2) and brain structure volumes. Each of these individual CSF neuroinflammatory markers was associated with the volume of specific brain regions (Table 4). In a separate binary regression model, the occurrence of NPS was associated with changes in volume in the hippocampus and ventricles (Nagelkerke $\mathrm{R}^{2}=0.525$; coefficients $=-8.459 *$ and $4.233^{\star *}$ respectively).

Table 4

Associations of volumetric data with cerebrospinal fluid neuroinflammatory marker concentration assessed with linear regression models.

\begin{tabular}{|lll|}
\hline Marker and associated regions & $R^{2}$ & Coeff. \\
\hline C-reactive protein & 0.146 \\
\hline Cerebellum & & $-5.561^{* \star}$ \\
\hline Pons & & $3.106^{*}$ \\
\hline 10 kDa IFN-y induced protein & 0.233 & \\
\hline Insula & & $-1.598^{* \star}$ \\
\hline Cerebellum & & $1.602^{* \star}$ \\
\hline Pallidum & & $1.110^{*}$ \\
\hline Soluble intracellular adhesion molecule-1 & 0.307 & \\
\hline Hippocampus & & $-0.862^{* \star}$ \\
\hline 3rd Ventricle & & $0.362^{* \star}$ \\
\hline
\end{tabular}

Table 4. Individual model $\mathrm{R}^{2}$ as well as $\beta$-coefficients for each significantly associated neuroinflammatory molecule are shown. ${ }^{*} P$ value $<.05 ; * * P$ value $<.01 ; * \star * ~ P$ value $<.001$

\subsection{NPS, neuroinflammation and cognitive decline}

In the whole cohort, the NPI-Q score was positively correlated with both CDR and CDR-SoB and negatively correlated with MMSE (Table 5). Binary logistic regression confirmed this association between NPI-Q score and cognitive status at baseline $\left(\right.$ Nagelkerke $\mathrm{R}^{2}=0.206$; coefficient $=0.278 ; P$ value $\left.=.004\right)$. When 
only considering participants with a positive NPI-Q score most individual NPS categories were correlated with cognitive measures (Table 5). We further correlated NPI-Q scores at baseline with cognitive decline as measured by changes in CDR-SoB at follow-up clinical assessments at 18 and 36 months $(n=87$ and 85 respectively, Fig. 3A) in the whole cohort and in participants with a positive NPI-Q score. We also tested in a linear regression model the associations between the concentrations of molecules identified in the CSF signature and cognitive decline. Only sICAM-1 was positively associated with cognitive decline at 18 and 36 months (Fig. 3B). A mediation model testing the interaction between NPI-Q score and sICAM-1 concentration revealed that the association between NPI-Q and CDR-SoB change at 18 months is mediated by sICAM-1 concentration (Fig. 3C).

Table 5

Correlations between NPI-Q score and cognitive assessment.

\begin{tabular}{|c|c|c|c|}
\hline & CDR & CDRSOB & MMSE \\
\hline NPI-Q score (whole cohort) & $0.406 * \star$ & $0.535^{\star \star}$ & $-0.428^{\star \star}$ \\
\hline NPI-Q score > 0 & 0.195 & $0.305^{\star}$ & -0.183 \\
\hline Delusions & 0.086 & $0.230^{\star}$ & -0.161 \\
\hline Hallucinations & 0.045 & 0.203 & $-0.219^{\star}$ \\
\hline Agitation & $0.373^{* \star}$ & $0.475^{* \star}$ & $-0.409^{\star \star}$ \\
\hline Depression/Dysphoria & $0.196^{*}$ & $0.265^{\star}$ & $-0.216^{*}$ \\
\hline Anxiety & $0.293^{* *}$ & $0.416^{* *}$ & $-0.346^{* \star}$ \\
\hline Apathy & -0.018 & 0.096 & -0.175 \\
\hline Irritability & $0.422^{* *}$ & $0.546^{* *}$ & $-0.449^{\star \star}$ \\
\hline Euphoria & 0.085 & $0.200^{*}$ & -0.150 \\
\hline Disinhibition & $0.226^{*}$ & $0.326^{* *}$ & $-0.273^{*}$ \\
\hline Aberrant motor behavior & $0.301^{\star *}$ & $0.314^{\star *}$ & $-0.375^{\star \star}$ \\
\hline Night-time behavior & 0.110 & 0.156 & -0.108 \\
\hline Appetite/Eating & $0.245^{*}$ & $0.313^{\star *}$ & $-0.266^{*}$ \\
\hline
\end{tabular}

\section{Table 5}

Correlations were assessed with Spearman's rho and one-tailed test of significance. ${ }^{*} P$ value $<.05 ; * \star P$ value $<.01$. NPI-Q, Neuropsychiatric inventory questionnaire score; CDR, Clinical Dementia Rating; CDRSoB, Clinical Dementia Rating Sum of Boxes; MMSE, Mini-Mental State Exam. 


\section{Discussion:}

We have identified combinations of inflammation molecules that best predict the occurrence of NPS in both CSF (CRP, IP-10, II-8 and sICAM-1) and serum (Eotaxin-3, IL-6 and CRP). Distinct marker combinations in CSF (TARC only) and serum (sICAM-1, IL-6 and IFN- $\gamma$ ) were associated with the severity of NPS. Individual NPS symptoms were associated with specific neuroinflammatory molecules in both CSF and serum. The identified CSF neuroinflammation molecules were associated with volume changes in specific brain regions. Finally, NPS were associated with more rapid cognitive decline at follow-up and this association was mediated by sICAM-1 CSF levels.

\subsection{Specific CSF neuroinflammatory molecules are associated with NPS}

Amongst the molecules related to NPS in our study, CRP and sICAM-1 have been previously associated with the presence of $A D$ pathology $(22,33)$. However, the association of these neuroinflammatory markers with NPS is to our knowledge novel. In this study, sICAM-1 in particular, is strongly associated with NPS. This association could be driven by the large number of participants with AD pathology in the NPI-Q > 0 group. However, accounting for the presence of cerebral core AD pathology does not alter the specific combination of molecules associated with NPS in CSF. Therefore, this association is at least partially independent of AD. In this signature, CRP, IP-10 and sICAM-1 could play different roles as high concentrations of CRP and IP-10 are associated with lower NPI-Q scores, suggesting a "neuroprotective" effect; while a higher concentration of sICAM-1 is associated with the presence of NPS, suggesting a deleterious role.

We have shown this CSF signature is independent of cognitive status suggesting neuroinflammation leading to NPS also occurs in the absence, or before the onset, of cognitive decline. Furthermore, this signature is not dependent on BBB function, suggesting that the related inflammatory process originates within the CNS. We have also shown that it is not only the absolute concentration of these molecules that is associated with NPI-Q score, but rather their relative concentrations between one another and their pattern of expression and interplay. For example, IP-10 does not display a different concentration between participants exhibiting NPS and those who do not, and does not correlate with total NPI-Q score either; it does however strongly associate with the occurrence of NPS when considering changes in CRP and sICAM-1 concentrations. This novel finding is in accordance with the concept that cytokines form a complex network enhancing and/or suppressing the production of each other (34).

\subsection{A distinct serum inflammation signature is associated with NPS}

We identified a distinct serum inflammatory signature of the occurrence of NPS containing Eotaxin-3, IL-6 and CRP. Unlike the CSF inflammatory marker profile, this signature is related to cognitive status, BBB function and the presence of cerebral core AD pathology. This confirms systemic inflammation may both contribute to predisposing to or enhancing CNS inflammation which may further result in cerebral dysfunction and neuronal injury, and the manifestation of both cognitive impairment and NPS (25). In has been previously shown that systemic inflammation may also reflect cerebral pathology (26) and 
neuroinflammation related to NPS (22). It is also plausible that following BBB breakdown, caused by AD or neuroinflammation (29), cross-talk between both CNS originating and circulating inflammation occurs (35) as is the case in aging (36). These data suggest a deleterious feedback loop where circulating neuroinflammatory molecules such as IL- 6 and IFN-y can further enhance expression of CNS inflammatory molecules (CRP and IP-10 respectively, $(37,38)$ ) that together can further damage the BBB (39) and activate microglia (40). The result is an escalation of neuroinflammation, further contributing to CNS processes leading to the manifestation of NPS. While we do not elucidate here the origin of these inflammatory processes, since subjects with manifest unstable medical conditions, including inflammation were excluded from the present study, we show these specific markers are particularly relevant for the occurrence of NPS.

\subsection{Specific mechanisms relate to the severity of NPS}

Distinct molecules, with the exception of sICAM-1 were associated with the severity of NPS both in CSF and serum in our study. This suggests that besides the identified inflammatory processes associated with the appearance of NPS, additional mechanisms may modulate their extent and severity. We therefore suggest that CNS inflammation and BBB breakdown could trigger the appearance of NPS. Following these events, CSF TARC along with circulating, IL-6, IFN-y, MCP-4 and IL-16, could regulate and enhance existing symptoms or play a regulatory role in the inflammatory response and dictate specific and localized responses.

\subsection{Individual NPS symptoms have specific pathological mechanisms}

Several individual NPS symptoms were associated to specific neuroinflammatory molecule signatures in CSF and serum. Amongst these, the strongest associations were found with the CSF signatures of Anxiety and Disinhibition and the serum signature of Depression. Symptom specific profiles differed between CSF and serum in all cases. Some of the molecules involved in these signatures (CRP, sICAM-1, IP-10, VEGF) are part of the signature of overall presence of NPS.

Previous evidence suggests neuroinflammation can lead to a variety of NPS (21). We however describe novel and specific associations of the identified molecules, such as sICAM-1 and VEGF-D, with multiple $\mathrm{NPI}-\mathrm{Q}$ categories suggesting that these symptoms may have common pathogenic mechanisms involving the associated molecule. In particular, higher CSF sICAM-1 levels were positively associated with symptoms of Depression, Anxiety, Apathy and Disinhibition in our cohort and could therefore play a role in the pathogenesis of all these symptom. We also found concentrations of VEGF-D in serum to be associated with the occurrence of Agitation, Depression, and Night-time behavior disorders. Furthermore, serum VEGF-D was associated with the occurrence of NPS only in the presence of cerebral AD. This suggests a role for this molecule in the pathogenesis of these NPS in the specific context of AD.

Contrary to these molecules, some of the identified molecules were associated with only a single symptom category; some of which have previously been reported, such as serum IP-10 with sleep disturbances (41) and serum IL-8 with Anxiety (42). The associations of MCP-4 with Agitation and CSF 
MDC with Night-time behavior however are novel. Overall, these findings suggest that while neuroinflammation results in a wide spectrum of neuropsychiatric manifestations, specific neuroinflammatory mediators could play a more important role in certain single syndromes.

\subsection{Brain regions associated with individual NPS symptoms}

The molecules we identified as part of the CSF neuroinflammatory profiles related to NPS have previously been associated with volume changes in specific brain regions (43-45), together suggesting neurodegeneration associated with neuroinflammation within these individual regions. We also observed that the volume of specific brain regions is associated with the concentration of neuroinflammatory markers.

The strong association of sICAM- 1 with the volume of the hippocampus and $3^{\text {rd }}$ ventricle (reflecting atrophy of surrounding areas) suggests these regions may play a role in the pathogenesis of NPS, although we cannot exclude sICAM-1 is linked to NPS via mechanisms independent of atrophy. Interestingly, these regions have been associated with the progression of $A D$ pathology and inflammation in previous work $(46,47)$. Considering our observations and previous reports $(13)$, we infer that decreased volume of the hippocampus and increased $3^{\text {rd }}$ ventricle volume may indicate regional neurodegeneration and neuroinflammation together involved in the pathogenesis of NPS of the symptoms associated with sICAM-1 (i.e. Anxiety, Depression and Disinhibition).

\subsection{NPS, neuroinflammation and clinical disease progression}

While the occurrence of NPS is associated with the presence of AD pathology, we have shown that the CSF neuroinflammatory signature of NPS is independent of AD. This finding suggests that at CNS level both $A D$ core pathology and neuroinflammatory processes may engage similar pathways leading to NPS. CSF sICAM-1 was previously found to correlate with both tau and pTau181 levels, confirming it is involved in AD-related tau-pathology and neural injury (22). In our models, the interaction between the presence of $A D$ pathology and SICAM-1 was significant, suggesting that SICAM-1 is present in both the $A D$ and neuroinflammation pathways related to NPS.

Higher NPS severity was associated with more rapid cognitive decline. This is in line with previous research describing an association of NPS with more rapid clinical disease progression in $\operatorname{AD}(4,10,9)$. The sICAM-1 CSF concentration is also associated with cognitive decline and our interaction analysis suggests that the association between NPS and cognitive decline is mediated by increased SICAM-1 in the CNS. A possible underlying mechanism is through altered cerebrovascular reactivity effects with which ICAM molecules are associated (48) which in turn, are known to differ according to cognitive status (49).

\subsection{Strengths and limitations}


Subjects included in this study had no psychiatric affection or symptom that could interfere with cognition and candidates with more marked neuropsychiatric symptoms were not considered. Consequently, there was a low frequency of some of the single NPS symptoms such as delusions and hallucinations in this cohort which does not allow to address possible relationships between inflammation and these symptoms. Whether the identified signatures may be used in clinical practice as markers of neuroinflammation-related NPS and targets for intervention needs to be further investigated in independent samples. Strengths of this study are the inclusion of elderly subjects with normal cognition or cognitive decline and the assessment of a large panel of inflammatory markers in paired serum and CSF samples. Furthermore, we addressed the relationships of inflammation related NPS with the core cerebral AD pathology, regional brain atrophy, and cognitive decline over time.

\subsection{Conclusion}

We have identified specific CSF and serum inflammatory signatures associated with NPS that can be considered both contributors to the underlying cerebral pathology and potential biomarkers of NPS. While the CSF signature identified here appears to indicate inflammatory processes that originate within the CNS and interact with the core AD pathology, the serum signature may represent systemic dysregulation of inflammatory activity related to BBB function and impacting CNS processes that lead to NPS. The inflammatory signatures of NPS indicate symptom specific underlying processes opening the perspective of targeted interventions to reduce NPS and their long-term consequences.

\section{Abbreviations}

Aß1-42 beta-amyloid 1-42

$A D$

AUC

BBB

CDR

CDR-SoB

CRP

CSF

CNS

IFN-Y

IL Interleukin
Alzheimer's Disease

area under the curve

Blood-brain barrier

Clinical Dementia Rating

CDR sum of boxes

C-reactive protein

Cerebrospinal fluid

Central Nervous System

Interferon- $\gamma$

Page 16/27 


$\begin{array}{ll}\text { IP-10 } & \text { 10 kDa interferon-y-induced protein } \\ \text { MCP-1,4 } & \text { monocyte chemoattractant protein 1,4 } \\ \text { MDC } & \text { macrophage-derived chemokine } \\ \text { MIP-1a } & \text { macrophage inflammatory protein 1a } \\ \text { MMSE } & \text { Mini-Mental State Examination } \\ \text { NPI-Q } & \text { Neuropsychiatric Inventory Questionnaire } \\ \text { NPS } & \text { Neuropsychiaric Symptoms } \\ \text { pTau181 } & \text { tau phosphorylated at threonine 181 } \\ \text { Qalb } & \text { CSF albumin index } \\ \text { ROC } & \text { receiver operating characteristic } \\ \text { SFLT-1 } & \text { soluble fms-like tyrosine kinase-1 } \\ \text { SICAM-1 } & \text { soluble intracellular cell adhesion molecule-1 } \\ \text { TARC } & \text { thymus and activation-regulated chemokine } \\ \text { Tau } & \text { total-tau } \\ \text { VEGF } & \text { vascular endothelial growth factor } \\ \text { VEGF-D } & \text { vascular endothelial growth factor D precursor } \\ \text { VIF } & \text { variance inflation factor }\end{array}$

\section{Declarations}

\section{Ethics declaration and consent of participants:}

The local ethics committee of canton Vaud (Switzerland) approved this study (No. 171/2013), and all participants or their legal representatives provided written informed consent.

\section{Availability of data:}

The datasets used and/or analysed during the current study are available from the corresponding author on reasonable request.

\section{Disclosures:}


JP received consultation honoraria from Nestle Institute of Health Sciences, Innovation Campus, EPFL, Lausanne, Switzerland, Ono Pharma, and from Fujirebio Europe. The other authors declare no potential conflicts of interest

\section{Funding:}

This work was supported by grants from the Swiss National Research Foundation (to JP, SNF 320030_141179), Synapsis Foundation - Alzheimer Research Switzerland (to JP, grant number 2017PI01) and funding from the Nestlé Institute of Health Sciences.

\section{Authors' contributions:}

C.C and J.P contributed to study design. C.C wrote the article, performed data modeling, statistical analyses, data interpretation and made figures. J.R. and B.M. provided regional volumetric data. G.B and L.D provided neuroinflammatory biomarker assessment. J.P contributed APOE genotype assessment and AD CSF biomarker assessment, contributed to writing the article and provided data interpretation and a critical review of the whole manuscript. All authors read, reviewed and approved the final manuscript.

\section{Acknowledgements:}

The authors wish to thank Richard Kirkland from Prometheus Laboratories for performing measurements and Dr Cornelia Pocnet and Dr Leonardo Zullo from University Hospital Geneva for their comments and critical assessment of the manuscript.

\section{References}

1. Ballard C, Corbett A. Management of neuropsychiatric symptoms in people with dementia. CNS Drugs 2010; 24(9):729-39.

2. Murphy R, O'Donoghue S, Counihan T, McDonald C, Calabresi PA, Ahmed MA et al. Neuropsychiatric syndromes of multiple sclerosis. Journal of neurology, neurosurgery, and psychiatry 2017; 88(8):697-708.

3. Youn JC, Lee DY, Jhoo JH, Kim KW, Choo IH, Woo Jl. Prevalence of neuropsychiatric syndromes in Alzheimer's disease (AD). Archives of gerontology and geriatrics 2011; 52(3):258-63.

4. Rozum WJ, Cooley B, Vernon E, Matyi J, Tschanz JT. Neuropsychiatric symptoms in severe dementia: Associations with specific cognitive domains the Cache County Dementia Progression Study. International journal of geriatric psychiatry 2019.

5. Apostolova LG, Cummings JL. Neuropsychiatric manifestations in mild cognitive impairment: a systematic review of the literature. Dementia and geriatric cognitive disorders 2008; 25(2):115-26.

6. Diniz BS, Butters MA, Albert SM, Dew MA, Reynolds, C. F., 3rd. Late-life depression and risk of vascular dementia and Alzheimer's disease: systematic review and meta-analysis of community- 
based cohort studies. The British journal of psychiatry : the journal of mental science 2013; 202(5):329-35.

7. Wise EA, Rosenberg PB, Lyketsos CG, Leoutsakos JM. Time course of neuropsychiatric symptoms and cognitive diagnosis in National Alzheimer's Coordinating Centers volunteers. Alzheimer's \& dementia (Amsterdam, Netherlands) 2019; 11:333-9.

8. Burhanullah MH, Tschanz JT, Peters ME, Leoutsakos JM, Matyi J, Lyketsos CG et al. Neuropsychiatric Symptoms as Risk Factors for Cognitive Decline in Clinically Normal Older Adults: The Cache County Study. The American journal of geriatric psychiatry : official journal of the American Association for Geriatric Psychiatry 2019.

9. Vetrano DL, Rizzuto D, Calderon-Larranaga A, Onder G, Welmer AK, Bernabei R et al. Trajectories of functional decline in older adults with neuropsychiatric and cardiovascular multimorbidity: A Swedish cohort study. PLoS medicine 2018; 15(3):e1002503.

10. Missotten P, Squelard G, Ylieff M, Di Notte D, Paquay L, Lepeleire J de et al. Relationship between quality of life and cognitive decline in dementia. Dementia and geriatric cognitive disorders 2008; 25(6):564-72.

11. McClam TD, Marano CM, Rosenberg PB, Lyketsos CG. Interventions for Neuropsychiatric Symptoms in Neurocognitive Impairment Due to Alzheimer's Disease: A Review of the Literature. Harvard review of psychiatry $2015 ; 23(5): 377-93$.

12. Kales HC, Gitlin LN, Lyketsos CG. Assessment and management of behavioral and psychological symptoms of dementia. BMJ (Clinical research ed.) 2015; 350:h369.

13. Boublay N, Schott AM, Krolak-Salmon P. Neuroimaging correlates of neuropsychiatric symptoms in Alzheimer's disease: a review of 20 years of research. European journal of neurology 2016; 23(10):1500-9.

14. Whitwell JL. Progression of atrophy in Alzheimer's disease and related disorders. Neurotoxicity research 2010; 18(3-4):339-46.

15. Dantzer R. Cytokine, sickness behavior, and depression. Immunol Allergy Clin North Am 2009; 29(2):247-64.

16. Combrinck MI, Perry VH, Cunningham C. Peripheral infection evokes exaggerated sickness behaviour in pre-clinical murine prion disease. Neuroscience 2002; 112(1):7-11.

17. Cunningham C, Campion S, Lunnon K, Murray CL, Woods JF, Deacon RM et al. Systemic inflammation induces acute behavioral and cognitive changes and accelerates neurodegenerative disease. Biol Psychiatry 2009; 65(4):304-12.

18. Cunningham C, Wilcockson DC, Campion S, Lunnon K, Perry VH. Central and systemic endotoxin challenges exacerbate the local inflammatory response and increase neuronal death during chronic neurodegeneration. J Neurosci 2005; 25(40):9275-84.

19. van Dooren FE, Schram MT, Schalkwijk CG, Stehouwer CD, Henry RM, Dagnelie PC et al. Associations of low grade inflammation and endothelial dysfunction with depression - The Maastricht Study. Brain, behavior, and immunity 2016; 56:390-6. 
20. Motivala SJ, Sarfatti A, Olmos L, Irwin MR. Inflammatory markers and sleep disturbance in major depression. Psychosomatic medicine 2005; 67(2):187-94.

21. Capuron L, Castanon N. Role of Inflammation in the Development of Neuropsychiatric Symptom Domains: Evidence and Mechanisms. Current topics in behavioral neurosciences 2017; 31:31-44.

22. Popp J, Oikonomidi A, Tautvydaite D, Dayon L, Bacher M, Migliavacca E et al. Markers of neuroinflammation associated with Alzheimer's disease pathology in older adults. Brain, behavior, and immunity $2017 ; 62: 203-11$.

23. Oikonomidi A, Tautvydaite D, Gholamrezaee MM, Henry H, Bacher M, Popp J. Macrophage Migration Inhibitory Factor is Associated with Biomarkers of Alzheimer's Disease Pathology and Predicts Cognitive Decline in Mild Cognitive Impairment and Mild Dementia. Journal of Alzheimer's disease : JAD 2017; 60(1):273-81.

24. Holmes C, Cunningham C, Zotova E, Woolford J, Dean C, Kerr S et al. Systemic inflammation and disease progression in Alzheimer disease. Neurology 2009; 73(10):768-74.

25. Popp J. Delirium and cognitive decline: more than a coincidence. Current opinion in neurology 2013; 26(6):634-9.

26. Morgan AR, Touchard S, Leckey C, O'Hagan C, Nevado-Holgado AJ, Barkhof F et al. Inflammatory biomarkers in Alzheimer's disease plasma. Alzheimer's \& dementia : the journal of the Alzheimer's Association 2019.

27. Licastro F, Pedrini S, Caputo L, Annoni G, Davis LJ, Ferri C et al. Increased plasma levels of interleukin-1, interleukin-6 and alpha-1-antichymotrypsin in patients with Alzheimer's disease: peripheral inflammation or signals from the brain? J Neuroimmunol 2000; 103(1):97-102.

28. Cummings JL, Mega M, Gray K, Rosenberg-Thompson S, Carusi DA, Gornbein J. The Neuropsychiatric Inventory: comprehensive assessment of psychopathology in dementia. Neurology 1994; 44(12):2308-14.

29. Bowman GL, Dayon L, Kirkland R, Wojcik J, Peyratout G, Severin IC et al. Blood-brain barrier breakdown, neuroinflammation, and cognitive decline in older adults. Alzheimer's \& dementia : the journal of the Alzheimer's Association 2018; 14(12):1640-50.

30. ADNI Study Documents [cited Accessed 2020 Apr 27]. Available from: URL: http://adni.loni.usc.edu/methods/documents/.

31. Schmitter D, Roche A, Marechal B, Ribes D, Abdulkadir A, Bach-Cuadra M et al. An evaluation of volume-based morphometry for prediction of mild cognitive impairment and Alzheimer's disease. Neurolmage. Clinical 2015; 7:7-17.

32. Mortamet B, Bernstein MA, Jack, C. R., Jr., Gunter JL, Ward C, Britson PJ et al. Automatic quality assessment in structural brain magnetic resonance imaging. Magnetic resonance in medicine 2009; 62(2):365-72.

33. Ray S, Britschgi M, Herbert C, Takeda-Uchimura Y, Boxer A, Blennow K et al. Classification and prediction of clinical Alzheimer's diagnosis based on plasma signaling proteins. Nature medicine 2007; 13(11):1359-62. 
34. Becher B, Spath S, Goverman J. Cytokine networks in neuroinflammation. Nature reviews. Immunology 2017; 17(1):49-59.

35. Dayon L, Cominetti O, Wojcik J, Galindo AN, Oikonomidi A, Henry H et al. Proteomes of Paired Human Cerebrospinal Fluid and Plasma: Relation to Blood-Brain Barrier Permeability in Older Adults. Journal of proteome research 2019; 18(3):1162-74.

36. Yousef H, Czupalla CJ, Lee D, Chen MB, Burke AN, Zera KA et al. Aged blood impairs hippocampal neural precursor activity and activates microglia via brain endothelial cell VCAM1. Nature medicine 2019; 25(6):988-1000.

37. Ridker PM. C-reactive protein: eighty years from discovery to emergence as a major risk marker for cardiovascular disease. Clinical chemistry 2009; 55(2):209-15.

38. Luster AD, Unkeless JC, Ravetch JV. Gamma-interferon transcriptionally regulates an early-response gene containing homology to platelet proteins. Nature 1985; 315(6021):672-6.

39. Closhen D, Bender B, Luhmann HJ, Kuhlmann CR. CRP-induced levels of oxidative stress are higher in brain than aortic endothelial cells. Cytokine 2010; 50(2):117-20.

40. Clarner T, Janssen K, Nellessen L, Stangel M, Skripuletz T, Krauspe B et al. CXCL10 triggers early microglial activation in the cuprizone model. Journal of immunology (Baltimore, Md. : 1950) 2015; 194(7):3400-13.

41. Huang WY, Huang CC, Chang CC, Kor CT, Chen TY, Wu HM. Associations of Self-Reported Sleep Quality with Circulating Interferon Gamma-Inducible Protein 10, Interleukin 6, and High-Sensitivity CReactive Protein in Healthy Menopausal Women. PloS one 2017; 12(1):e0169216.

42. Janelidze S, Suchankova P, Ekman A, Erhardt S, Sellgren C, Samuelsson M et al. Low IL-8 is associated with anxiety in suicidal patients: genetic variation and decreased protein levels. Acta psychiatrica Scandinavica 2015; 131(4):269-78.

43. Han JH, Wong KS, Wang YY, Fu JH, Ding D, Hong Z. Plasma level of sICAM-1 is associated with the extent of white matter lesion among asymptomatic elderly subjects. Clinical neurology and neurosurgery 2009; 111(10):847-51.

44. Satizabal CL, Zhu YC, Mazoyer B, Dufouil C, Tzourio C. Circulating IL-6 and CRP are associated with MRI findings in the elderly: the 3C-Dijon Study. Neurology 2012; 78(10):720-7.

45. Gongvatana A, Correia S, Dunsiger S, Gauthier L, Devlin KN, Ross S et al. Plasma cytokine levels are related to brain volumes in HIV-infected individuals. Journal of neuroimmune pharmacology : the official journal of the Society on Neurolmmune Pharmacology 2014; 9(5):740-50.

46. Barron AM, Tokunaga M, Zhang MR, Ji B, Suhara T, Higuchi M. Assessment of neuroinflammation in a mouse model of obesity and beta-amyloidosis using PET. Journal of neuroinflammation 2016; 13(1):221.

47. Bartos A, Gregus D, Ibrahim I, Tintera J. Brain volumes and their ratios in Alzheimer s disease on magnetic resonance imaging segmented using Freesurfer 6.0. Psychiatry research. Neuroimaging $2019 ; 287: 70-4$. 
48. Novak V, Zhao P, Manor B, Sejdic E, Alsop D, Abduljalil A et al. Adhesion molecules, altered vasoreactivity, and brain atrophy in type 2 diabetes. Diabetes care 2011;34(11):2438-41.

49. Richiardi J, Monsch AU, Haas T, Barkhof F, van de Ville D, Radu EW et al. Altered cerebrovascular reactivity velocity in mild cognitive impairment and Alzheimer's disease. Neurobiology of aging 2015; 36(1):33-41.

\section{Figures}


Clark - Neuroinflammation and neuropsychiatric symptoms

A

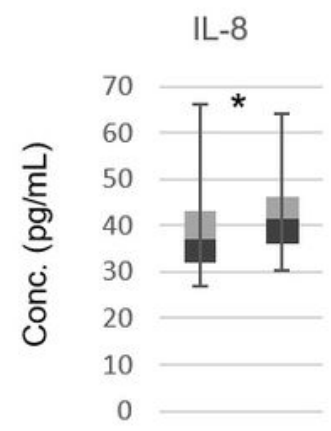

B

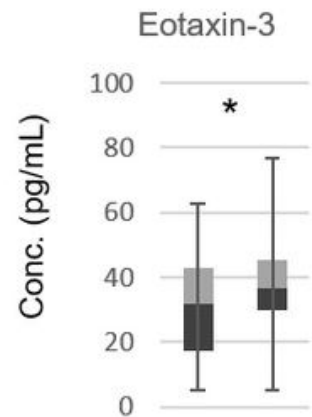

C

CSF markers - NPI-Q score

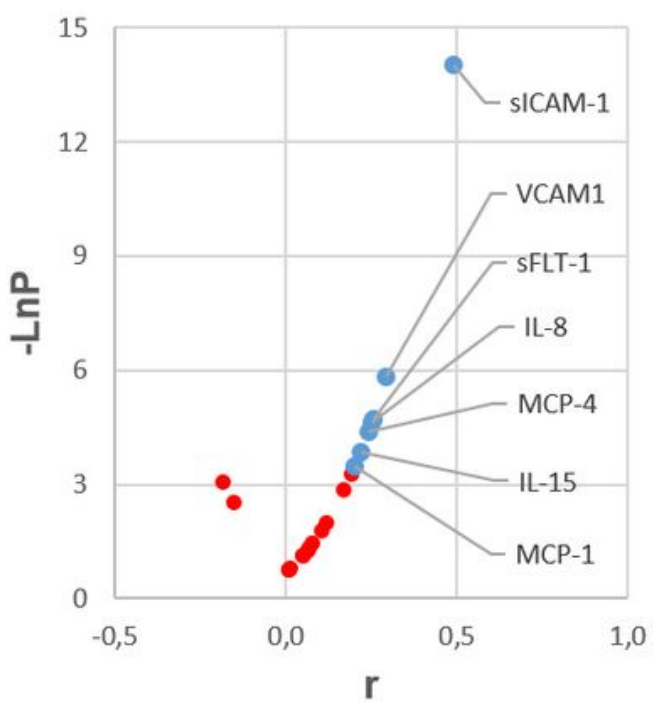

IL-15

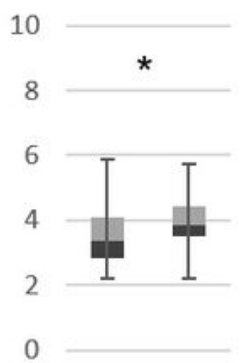

MIP-1a

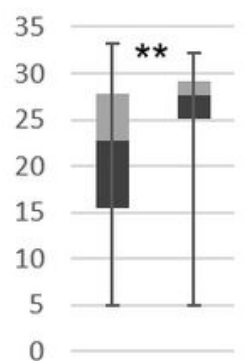

sICAM-1

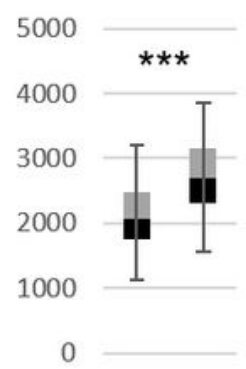

sVCAM-1

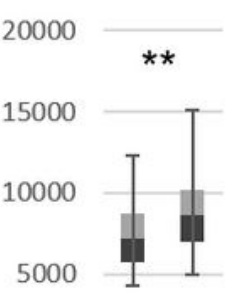

0

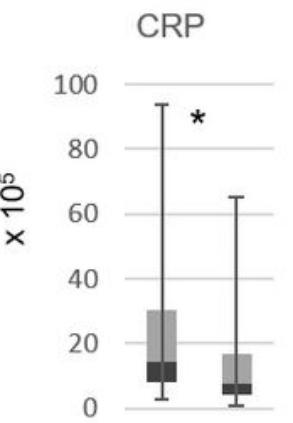

\section{D}

Serum markers - NPI-Q score

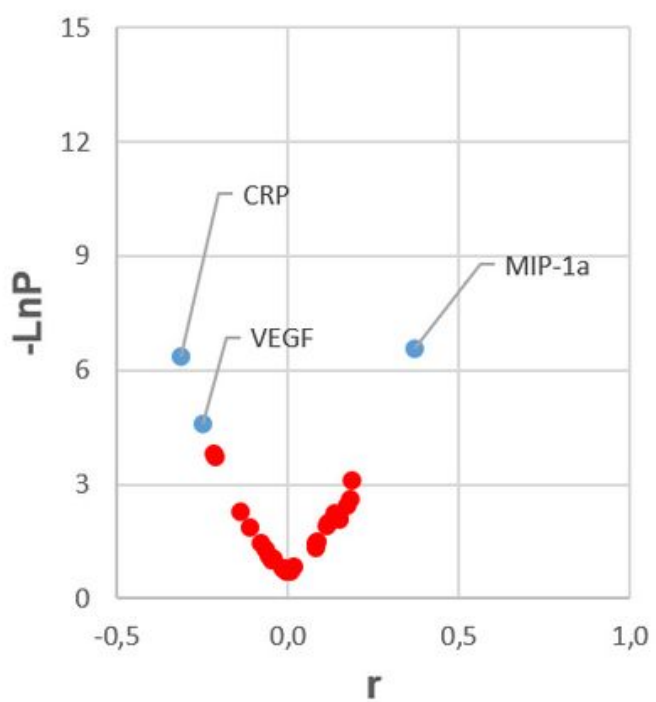

Figure 1

\section{Figure 1}

Concentrations and correlations of neuroinflammatory markers in cerebrospinal fluid (CSF) and Serum with Neuropsychiatric inventory questionnaire (NPI-Q) score in whole cohort. A-B) Box plots of molecules showing a significantly different concentration between control (left) participants and those exhibiting neuropsychiatric symptoms (right) in CSF (A) and serum (B). C-D) Correlation of CSF(C) and serum (D) markers with NPI-Q score. Significantly correlated markers after correction for multiple testing are shown 
in blue and labelled on the graph. IL-8, Interleukin-8; IL-15, Interleukin-15; sFLT-1, soluble fms-like tyrosine kinase-1; sICAM-1, soluble intracellular adhesion molecule-1; sVCAM-1, circulating vascular cell adhesion molecule-1; MIP-1a, macrophage Inflammatory protein 1a; CRP, C-reactive protein; MCP-1(4), monocyte chemoattractant protein 1(4); VEGF, vascular endothelial growth factor; * $P$ value < .05; ** $P$ value $<.01$; $\star \star \star P$ value $<.001 ;-L n P$, inverse logarithm of $P$ value; $r$ Spearman's rho.

Clark - Neuroinflammation and neuropsychiatric symptoms

A

\begin{tabular}{|l|l|l|l|l|l|l|l|l|}
\hline Model & \multicolumn{2}{|c}{ Uncorrected } & \multicolumn{2}{c}{ CDR } & \multicolumn{2}{c|}{ CSF AD } & \multicolumn{2}{c|}{ BBB } \\
\hline $\mathbf{R}^{2}$ & \multicolumn{2}{|c|}{$\mathbf{0 . 5 1 0}$} & \multicolumn{2}{c|}{$\mathbf{0 . 5 2 9}$} & \multicolumn{2}{c|}{0.571} & \multicolumn{2}{c|}{0.482} \\
\hline Marker & Coeff. & $\boldsymbol{P}$ value & Coeff. & $\boldsymbol{P}$ value & Coeff. & $\boldsymbol{P}$ value & Coeff. & $\boldsymbol{P}$ value \\
\hline CRP & -0.845 & 0.007 & -0.830 & 0.01 & -0.682 & 0.049 & -0.771 & 0.011 \\
\hline IP10 & -2.527 & 0.004 & -2.428 & 0.007 & -2.654 & 0.005 & -1.756 & 0.017 \\
\hline sICAM-1 & 6.217 & $<0.001$ & 5.728 & $<0.001$ & 5.438 & $<0.001$ & 5.243 & 0.001 \\
\hline IL-8 & 3.612 & 0.026 & 3.259 & 0.05 & 4.116 & 0.021 & NS & NS \\
\hline AD status & N/A & N/A & N/A & N/A & 1.626 & 0.013 & N/A & N/A \\
\hline
\end{tabular}

$\mathrm{B}$

\begin{tabular}{|l|l|l|l|l|l|l|l|l|}
\hline Model & \multicolumn{2}{|c|}{ Uncorrected } & \multicolumn{2}{c|}{ CDR } & \multicolumn{2}{c|}{ CSF AD } & \multicolumn{2}{c|}{ BBB } \\
\hline$R^{2}$ & \multicolumn{2}{|c|}{0.307} & \multicolumn{2}{c|}{0.304} & \multicolumn{2}{c|}{0.320} & \multicolumn{2}{c|}{0.393} \\
\hline Marker & Coeff. & $\boldsymbol{P}$ value & Coeff. & $\boldsymbol{P}$ value & Coeff. & $\boldsymbol{P}$ value & Coeff. & $\boldsymbol{P}$ value \\
\hline CRP & -1.184 & 0.05 & $-0.752^{*}$ & 0.042 & -0.784 & 0.033 & NS & NS \\
\hline Eotaxin-3 & 1.074 & 0.046 & NS & NS & NS & NS & NS & NS \\
\hline IL-6 & 1.257 & 0.026 & NS & NS & NS & NS & NS & NS \\
\hline VEGF-D & NS & NS & NS & NS & -2.22 & 0.044 & NS & NS \\
\hline MIP-1a & NS & NS & NS & NS & NS & NS & 1.665 & 0.28 \\
\hline VEGF & NS & NS & NS & NS & NS & NS & -0.867 & 0.42 \\
\hline Qalb & N/A & N/A & N/A & N/A & N/A & N/A & 0.429 & 0.002 \\
\hline AD status & N/A & N/A & N/A & N/A & 1.890 & 0.008 & N/A & N/A \\
\hline
\end{tabular}

C

\begin{tabular}{|l|l|l|l|l|l|l|l|l|}
\hline Model & \multicolumn{2}{|c|}{ Uncorrected } & \multicolumn{2}{c|}{ CDR } & \multicolumn{2}{c|}{ CSF AD } & \multicolumn{2}{c|}{ BBB } \\
\hline $\mathbf{R}^{2}$ & \multicolumn{2}{|c|}{0.268} & \multicolumn{2}{c|}{0.281} & \multicolumn{2}{c|}{0.269} & \multicolumn{2}{c|}{0.269} \\
\hline Marker & Coeff. & $\boldsymbol{P}$ value & Coeff. & $\boldsymbol{P}$ value & Coeff. & $\boldsymbol{P}$ value & Coeff. & $\boldsymbol{P}$ value \\
\hline TARC & 0.518 & 0.001 & 0.494 & 0.002 & 0.517 & 0.001 & 0.516 & 0.001 \\
\hline
\end{tabular}

D

\begin{tabular}{|l|l|l|l|l|l|l|l|l|}
\hline Model & \multicolumn{2}{|c|}{ Uncorrected } & \multicolumn{2}{c|}{ CDR } & \multicolumn{2}{c|}{ CSF AD } & \multicolumn{2}{c|}{ BBB } \\
\hline R $^{2}$ & \multicolumn{2}{|c|}{$\mathbf{0 . 7 0 7}$} & \multicolumn{2}{c|}{0.310} & \multicolumn{2}{c|}{0.767} & \multicolumn{2}{c|}{0.706} \\
\hline Marker & Coeff. & $\boldsymbol{P}$ value & Coeff. & $\boldsymbol{P}$ value & Coeff. & $\boldsymbol{P}$ value & Coeff. & $\boldsymbol{P}$ value \\
\hline IL-6 & 0.568 & $<0.001$ & 0.552 & 0.007 & 0.567 & $<0.001$ & 0.507 & 0.001 \\
\hline SICAM-1 & 0.617 & $<0.001$ & NS & NS & 0.649 & $<0.001$ & 0.722 & $<0.001$ \\
\hline IFN-V & -0.686 & $<0.001$ & NS & NS & -0.705 & $<0.001$ & -0.700 & $<0.001$ \\
\hline MCP4 & 0.374 & 0.15 & NS & NS & 0.373 & 0.017 & 0.386 & 0.009 \\
\hline IL-16 & NS & NS & NS & NS & -0.254 & 0.047 & -0.251 & 0.042 \\
\hline
\end{tabular}

Figure 2

Figure 2 
Regression models revealing associations between neuroinflammatory molecules and neuropsychiatric symptoms (NPS). A-B) Associations of cerebrospinal fluid (A) and serum (B) neuroinflammatory marker concentrations with the occurrence of NPS. C-D) Associations of CSF (C) and serum (D) neuroinflammatory marker concentrations with the severity of NPS. Results from binary logistic (A and B) and linear ( $C$ and $D$ ) regressions uncorrected, corrected for cognitive status (CDR), corrected for the presence of Alzheimer's disease pathology (CSF AD) and corrected for blood-brain barrier (BBB) permeability are shown. Individual model Nagelkerke R2 ( $A$ and B) and R2 ( $C$ and D) are shown as well as (standardized for $C$ and $D$ ) $\beta$-coefficients for each significantly associated neuroinflammatory molecule. NS, not significant; N/A, not applicable; * $\mathrm{P}$ value <.05; ** $\mathrm{P}$ value $<.01$; $* \star \star ~ P$ value $<.001$; CRP, C-reactive protein; IP-10, 10 kDa IFN-y induced protein; sICAM-1, soluble intracellular adhesion molecule-1; IL-8, Interleukin-8; AD status, pTau/Aß1-42 ratio; IL-6, Interleukin-6, VEGF(-D), vascular endothelial growth factor (D precursor); MIP-1 a, macrophage inflammatory protein 1a; Qalb, Quotient albumin or CSF albumin index; TARC, thymus and activation-regulated chemokine; IL-6, Interleukin-6; IFN- $\gamma$, Interferon- $\gamma$; MCP4, monocyte chemoattractant protein 4; IL-16, Interleukin-16. 


\section{Clark - Neuroinflammation and neuropsychiatric symptoms}

\section{A}

Whole cohort

CDR decline 18 months CDR decline $\mathbf{3 6}$ months NPI-Q score $>0$ $0.332^{* *}$ $0.605^{\text {** }}$

0.185

$0.368^{*}$

\section{B}

\begin{tabular}{|l|l|l|l|l|l|l|}
\hline & \multicolumn{2}{|c|}{ Baseline } & \multicolumn{2}{c|}{ 18 months } & \multicolumn{2}{c|}{ 36 months } \\
\hline $\mathbf{R}^{2}$ & \multicolumn{2}{|c|}{0.065} & \multicolumn{2}{c|}{0.278} & \multicolumn{2}{c|}{0.282} \\
\hline Marker & Coeff. & $\boldsymbol{P}$ value & Coeff. & $\boldsymbol{P}$ value & Coeff. & $\boldsymbol{P}$ value \\
\hline CRP & -0.026 & 0.785 & -0.031 & 0.735 & -0.172 & 0.078 \\
\hline sICAM-1 & 0.270 & 0.008 & 0.276 & 0.006 & 0.362 & 0.001 \\
\hline IP-10 & -0.029 & 0.764 & 0.058 & 0.530 & 0.052 & 0.603 \\
\hline
\end{tabular}

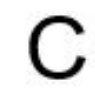

\begin{tabular}{|l|l|l|l|l|}
\hline & \multicolumn{2}{|c|}{18 months } & \multicolumn{2}{c|}{36 months } \\
\hline $\mathbf{R}^{2}$ & \multicolumn{2}{|c|}{0.313} & \multicolumn{2}{c|}{0.358} \\
\hline Marker & Coeff. & $\boldsymbol{P}$ value & Coeff. & $\boldsymbol{P}$ value \\
\hline NPI-Q & 5.75 & 0.98 & 3.073 & 0.549 \\
\hline sICAM-1 & 5.869 & 0.005 & 1.641 & 0.322 \\
\hline Interaction & -0.719 & 0.103 & -0.337 & $<0.520$ \\
\hline
\end{tabular}

\section{Figure 3}

Figure 3

Associations of neuroinflammatory markers with cognitive decline. A) Associations between neuroinflammatory markers and cognitive status at baseline and cognitive decline in the whole cohort measured by change in Clinical Dementia Rating Sum of Boxes at 18 and 36 months. Standardized $\beta-$ coefficients and R2 obtained by linear regression models are shown. B) Mediation model evaluating the contribution of NPI-Q score, sICAM-1 at baseline and their interaction to cognitive decline at 18 and 36 months. $\beta$-coefficients and Nagelkerke R2 obtained by binary logistic regression models are shown. CRP, C-reactive protein; sICAM-1, soluble intracellular molecule-1; IP-10, 10 kDa IFN-y induced protein, NPI-Q, Neuropsychiatric inventory questionnaire. 


\section{Supplementary Files}

This is a list of supplementary files associated with this preprint. Click to download.

- Supplementarytable3Clark.docx

- Supplementarytable1Clark.docx

- Supplementarytable2Clark.docx

- Supplementarytable4Clark.docx

- SupplementaryFigure1Clark.docx 